\title{
Open-Loop Beamforming Technique for MIMO System and Its Practical Realization
}

\author{
Peerapong Uthansakul, Apinya Innok, and Monthippa Uthansakul \\ School of Telecommunication Engineering, Suranaree University of Technology, Muang, Nakhon Ratchasima 30000, Thailand \\ Correspondence should be addressed to Apinya Innok, apinya_in@hotmail.com
}

Received 21 February 2011; Accepted 19 May 2011

Academic Editor: Miguel Ferrando

Copyright (C) 2011 Peerapong Uthansakul et al. This is an open access article distributed under the Creative Commons Attribution License, which permits unrestricted use, distribution, and reproduction in any medium, provided the original work is properly cited.

\begin{abstract}
The concept of close-loop beamforming for MIMO system was well known proposed the singular value decomposition on channel matrix. This technique can improve the capacity performance, but the cost of feedback channel and the complexity processing discard the interest of implementation. Therefore, this paper aims to investigate the benefit of using an open-loop beamforming for MIMO system in practical approaches. The low-profile concept of open-loop beamforming which is convenient for implementation is proposed by just inserting Butler matrices at both transmitter and receiver. The simulation and measurement results indicate that the open-loop beamforming with Butler matrix outperforms the conventional MIMO system. Although, the close-loop beamforming offers a better performance than open-loop beamforming technique, the proposed system is attractive because it is low cost, uncomplicated, and easy to implement.
\end{abstract}

\section{Introduction}

The MIMO (Multiple-input-multiple-output) system is a good quality of service such as channel capacity. In general, MIMO systems consideration of channel capacity is based on the array antennas at both transmitter and receiver.Many works have proposed the method of eigenbeamforming technique [1-5]. This technique utilizes the properties of estimated channels by performing singular value decomposition on channel matrix. Then eigenvectors compositing of channel matrix are considered as pre- and postcoding schemes for MIMO systems. This technique can improve the capacity performance, but both transmitter and receiver have to perfectly know the channel information, named as close loop MIMO system. However, there are many unattractive issues of using eigen-beamforming in practice such as a requirement of high system complexity and many procedures for channel feedback transmission. In turn, for open loop beamforming, the transmitter sends independent information symbols from multiple transmit antenna elements to the receiver. The received channels are not sent back to the transmitter. So, the transmitter does not know the channel information. The pre- and postcoding schemes do not require any additional complexity like close loop system. Therefore, the study of using open loop is focused on in this paper.

In the research areas of MIMO system, many works such as [6-9] have been proposed to enhance the channel capacity in order to satisfy the user demand for high data rate applications. Some studies have been focused on theoretical works and some have been performed by measurements. Nevertheless, most of them develop the technique to enhance the channel capacity through channel behavior [10-12] such as adjusting transmitted powers according to eigenvalue of channels as so-called water filling method. In general, it can be noticed that the theoretical consideration of channel capacity is based on the array antennas at both transmitter and receiver, but the channel characteristic is composed of many angle parameters such as angle of arrival, angle of departure, and angle spread. Therefore, it is interesting to investigate the performance of MIMO system using the open loop beamforming instead of the conventional MIMO system. Recently, the authors in [13, 14] developed the channel estimation of MIMO-OFDM system based on open loop beamforming consideration. The applicability 
of open loop beamforming technique is dependent on the channel stochastic information available to the receiver. The design of suitable pilots is proposed by facilitating the direct implementation of open loop beamforming and analyzing the performances of different channel estimation techniques. Although the significant improvement on MIMO capacity can be expected by using open loop beamforming, so far in the literature, there is no work to illustrate the capacity benefit of using open loop beamforming. The reason is that the pre- and postcoding schemes of angle transformations increase the complexity on both transmitter and receiver. Hence, it is challenging to find the technique with low cost and complexity matching with the concept of open loop beamforming. In [15], the proposed scheme uses a discrete fourier transformation (DFT) to receive a signal vector in RF domain. This can be realized by placing a Butler matrix between the antenna elements and the receiver switch. This paper presents only the simulations results. There is no measurement result presented in this paper. Clearly the difference between [15] and our paper is on the measurements. In [16], the authors investigate the correlation coefficients (line of sight and nonline of sight) via both simulation and measurement results. However, the analysis of correlation coefficients has not been analytically discussed yet. This is the claim of our novelty, that our paper presents the analytical analysis of correlation coefficients. Moreover, the additional measurements have been done by rotating the array direction for many degrees. These measurements are different from [16] and give more insight of using Butler matrix.We used the method of open loop beamforming in [17] investigated the capacity performance of compared with conventional MIMO system by computer simulations. The results in [17] confirm the advantages of using open loop beamforming. In this paper, the analytical analysis of how open loop beamforming impacts on the channel matrix is explained. Then, it gives a reason why using open loop beamforming for MIMO system provides a better performance over a conventional MIMO system. Also, further work from [17] is carried out by manufacturing a Butler matrix and performing the experimental results due to the fact that only simulation results cannot claim the use of the proposed system in practice. The reason why Butler matrix is chosen is because Butler matrix is just a low-complexity hardware that can offer the open loop beamforming. In general, there are infinite choices to choose the set of orthogonal steering vectors to form an open loop beamforming. Therefore, it is hard to justify whether Butler matrix provides the best performance among others. To focus on hardware complexity, the other methods to form open loop beamforming might need 16 phase shifters to simultaneously form 4 beams while Butler matrix approach uses only one low-cost printed circuit board. Thus, the authors construct the $4 \times 4$ MIMO system employing an open loop beamforming by Butler matrix which is a lowprofile concept and convenient for implementation. This matrix simultaneously forms multiple departure or arrival angles into four directions. By only inserting Butler matrix next to antenna arrays, the conventional MIMO systems can be transformed into the MIMO systems with open loop beamforming without the need of additional burden on processing units at both transmitter and receiver.

In summary, the contribution of this paper falls into three main issues. At first, the analytical analysis of how open loop beamforming impacts on the channel matrix is originally provided. This helps the reader to understand in a true benefit of open loop beamforming. Secondly, the practical realization of open loop beamforming for $4 \times 4$ MIMO systems has been demonstrated. The third contribution is on the experimental comparisons in terms of channel capacity. All contributions confirm either new concept or actual benefit of employing MIMO with open loop beamforming. The paper is organized as follows. In Section 2, the details of SISO system, conventional MIMO system, close loop beamforming, and open loop beamforming technique are described. Then in Section 3, the analytical analysis of using open loop beamforming is explained. The feature of Butler matrix to apply for open loop beamforming is given in Section 4. Section 5 describes the details of channel measurements. Section 6 provides the simulation and measurement results of open loop beamforming realized by Butler matrix in comparing with conventional MIMO system. Finally in Section 7, the conclusion of this paper is given.

\section{System Model}

2.1. SISO System. For a memoryless SISO (single-inputsingle-output) system, the Shannon capacity is given by [8]

$$
C=\log _{2}\left(1+\frac{P_{t} h}{P_{N}}\right),
$$

where $P_{t}$ is the transmitted power, $h$ is the wireless channel coefficient, and $P_{N}$ is the noise power in each branch of antennas at the receiver. Note that the signal-to-noise-power ratio (SNR) is defined as $P_{t} / P_{N}$.

2.2. Conventional MIMO System. We consider the narrowband MIMO channel. Let $\mathbf{x}$ be a vector of the transmitted signals with $N_{t}$ transmitted antennas and let $\mathbf{y}$ be a vector of the received signals with $N_{r}$ received antennas. Then the relation between transmitted and received signals is given by

$$
\mathbf{y}=\mathbf{H x}+\mathbf{n},
$$

where $\mathbf{n}$ is an $N_{r} \times 1$ noise vector and $\mathbf{H}$ is an $N_{r} \times N_{t}$ channel matrix. In this paper, the channel matrix is modelled by using the concept of geometrical two-ring model. This model is based on the extension of single-bounce two-ring scattering model for flat fading channels [19]. With this notation, channel output sequence can be written in matrix form as the following expressions:

$$
\left[\begin{array}{c}
y_{1} \\
y_{2} \\
\vdots \\
y_{N_{r}}
\end{array}\right]=\left[\begin{array}{cccc}
h_{11} & h_{12} & \cdots & h_{1 N_{t}} \\
h_{21} & h_{22} & \cdots & h_{2 N_{t}} \\
\vdots & \vdots & \ddots & \vdots \\
h_{N_{r} 1} & h_{N_{r} 2} & \cdots & h_{N_{r} N_{t}}
\end{array}\right]\left[\begin{array}{c}
x_{1} \\
x_{2} \\
\vdots \\
x_{N_{t}}
\end{array}\right]+\left[\begin{array}{c}
n_{1} \\
n_{2} \\
\vdots \\
n_{N_{r}}
\end{array}\right] .
$$


Figure 1 (a) shows the conventional $4 \times 4$ MIMO systems. There is an arbitrary number of physical paths between the transmitter and receiver [20]; the $i$ th path having attenuation of $a_{i}$, makes an angle of $\phi_{t i}\left(\Omega_{t i}:=\cos \phi_{t i}\right)$ with the transmit antenna array and angle of $\phi_{r i}\left(\Omega_{r i}:=\cos \phi_{r i}\right)$ with the receive antenna array. The channel matrix $\mathbf{H}$ can be written as the following expressions:

$$
\mathbf{H}=\sum_{i} a_{i}^{b} \mathbf{e}_{r}\left(\Omega_{r i}\right) \mathbf{e}_{t}\left(\Omega_{t i}\right)^{*},
$$

where

$$
\begin{gathered}
a_{i}^{b}:=a_{i} \sqrt{N_{t} N_{r}} \exp \left(-\frac{j 2 \pi d_{i}}{\lambda_{c}}\right), \\
\mathbf{e}_{t}(\Omega):=\frac{1}{\sqrt{N_{t}}}\left[\begin{array}{c}
1 \\
\exp \left[-j\left(2 \pi \Delta_{t} \Omega\right)\right] \\
\vdots \\
\exp \left[-j\left(N_{t}-1\right)\left(2 \pi \Delta_{t} \Omega\right)\right]
\end{array}\right], \\
\mathbf{e}_{r}(\Omega):=\frac{1}{\sqrt{N_{r}}}\left[\begin{array}{c}
\exp \left[-j\left(2 \pi \Delta_{r} \Omega\right)\right] \\
\vdots \\
\exp \left[-j\left(N_{r}-1\right)\left(2 \pi \Delta_{r} \Omega\right)\right]
\end{array}\right] .
\end{gathered}
$$

Also, $d_{i}$ is the distance between transmit and receive antennas along path $i$ th. Note that $(\cdot)^{*}$ is the conjugate and transpose operation. The vector $\mathbf{e}_{t}(\Omega)$ and $\mathbf{e}_{r}(\Omega)$ are, respectively, transmitted and received unit spatial signatures along the direction $\Omega, \lambda_{c}$ is the wavelength of the center frequency in a whole signal bandwidth. Assuming uniform linear array, the normalized separation between the transmit antennas is $\Delta_{t}$ (antenna separation $/ \lambda_{c}$ ) and the normalized separation between receive antennas is $\Delta_{r}$ (antenna separation $/ \lambda_{c}$ ). Note that the reason of normalization is because this proposed system can work in any frequency band. Hence, the normalization is made to neglect the unused parameter. When channel state information (CSI) is not available at the transmitter, the capacity of MIMO systems [21] expressed in bits per second per hertz $(\mathrm{bps} / \mathrm{Hz})$ can be written as

$$
C=\log _{2} \operatorname{det}\left(\mathbf{I}_{N_{r}}+\frac{P_{t}}{P_{N} N_{t}} \mathbf{H H}^{*}\right),
$$

where $\mathbf{I}_{N_{r}}$ is the identity matrix having $N_{r} \times N_{r}$ dimension, $\mathrm{H}$ is the channel matrix having $N_{r} \times N_{t}$ dimension with $\mathbf{H}^{*}$ being its transpose conjugate. In this paper, the channel matrix $\mathbf{H}$ is normalized by $\|\mathbf{H}\|_{F}^{2}=N_{r} N_{t}$.

2.3. MIMO System with Close Loop Beamforming Technique. Figure 1(b) showed the close loop beamforming representation of MIMO systems. We can refer to eigen-beamforming technique. Considering an MIMO channel with $N_{r} \times N_{t}$ channel matrix $\mathbf{H}$ that is known to both the transmitter and the receiver, the eigenvectors can be found by applying SVD technique to channel matrix as shown below:

$$
\mathbf{H}=\mathbf{W S V}^{*},
$$

where $N_{r} \times N_{r}$ matrix $\mathbf{W}$ and the $N_{t} \times N_{t}$ matrix $\mathbf{V}$ are unitary, and $\mathbf{S}$ is an $N_{r} \times N_{t}$ diagonal matrix. These two matrices are used as pre- and postcoding matrices at transmitter and receiver, respectively. So the channel matrix when applying pre and post matrices can be written as follows:

$$
\mathbf{H}^{e}=\mathbf{W}^{*} \mathbf{H V} .
$$

Thus, the capacity of MIMO system is given by

$$
C=\log _{2} \operatorname{det}\left(\mathbf{I}_{N_{r}}+\frac{P_{t}}{P_{N} N_{t}} \mathbf{H}^{e} \mathbf{H}^{e *}\right) .
$$

2.4. MIMO System with Open Loop Beamforming Technique. Figure 1(c) showed the open loop beamforming representation of MIMO systems. The open loop beamforming can be represented by the transmitted and received signals. The signal arriving at a directional cosine $\Omega$ into the receive antenna array is along the unit spatial signature $\mathbf{e}_{r}(\Omega)$ given by (7). Hence, the particularly fixed sequence of $\Omega$ can form a special set of $N_{r} \times 1$ vectors given as

$$
\xi_{r}:=\left\{\mathbf{e}_{r}(0), \mathbf{e}_{r}\left(\frac{1}{L_{r}}\right), \ldots, \mathbf{e}_{r}\left(\frac{N_{r}-1}{L_{r}}\right)\right\} .
$$

In (12), it can be noticed that there is a set of orthogonal basis for the received signal space. This basis provides the representation of received signals in the open loop beamforming.

It is similarly defined for the open loop beamforming representation of the transmitted signal. The signal transmitted at direction $\Omega$ is along the unit vector $\mathbf{e}_{t}(\Omega)$, defined in (6). The set of $N_{t} \times 1$ sequential vectors is given as

$$
\xi_{t}:=\left\{\mathbf{e}_{t}(0), \mathbf{e}_{t}\left(\frac{1}{L_{t}}\right), \ldots, \mathbf{e}_{t}\left(\frac{N_{t}-1}{L_{t}}\right)\right\},
$$

where $L_{t}=N_{t} \Delta_{t}$ and $L_{r}=N_{r} \Delta_{r}$ are the normalized antenna array lengths of the transmitter and receiver, respectively. Let $\mathbf{U}_{t}$ and $\mathbf{U}_{r}$ be the unitary matrices whose columns are the basis vector in (13) and (12), respectively, which can be written as

$$
\mathbf{U}_{t}=\frac{1}{\sqrt{N_{t}}}\left[\begin{array}{cccc}
1 & 1 & \cdots & 1 \\
1 & e^{\left(-j 2 \pi / N_{t}\right)} & \cdots & e^{\left(-j 2 \pi\left(N_{t}-1\right) / N_{t}\right)} \\
\vdots & \vdots & \ddots & \vdots \\
1 & e^{\left(-j 2 \pi\left(N_{t}-1\right) / N_{t}\right)} & \cdots & e^{\left(-j 2 \pi\left(N_{t}-1\right)\left(N_{t}-1\right) / N_{t}\right)}
\end{array}\right] \text {, }
$$

$$
\mathbf{U}_{r}=\frac{1}{\sqrt{N_{t}}}\left[\begin{array}{cccc}
1 & 1 & \cdots & 1 \\
1 & e^{\left(-j 2 \pi / N_{r}\right)} & \cdots & e^{\left(-j 2 \pi\left(N_{r}-1\right) / N_{r}\right)} \\
\vdots & \vdots & \ddots & \vdots \\
1 & e^{\left(-j 2 \pi\left(N_{r}-1\right) / N_{r}\right)} & \cdots & e^{\left(-j 2 \pi\left(N_{r}-1\right)\left(N_{r}-1\right) / N_{r}\right)}
\end{array}\right] .
$$

We can transform the conventional MIMO system into the open loop beamforming by the following expression:

$$
\mathbf{H}^{a}:=\mathbf{U}_{r}^{*} \mathbf{H U}_{t} .
$$




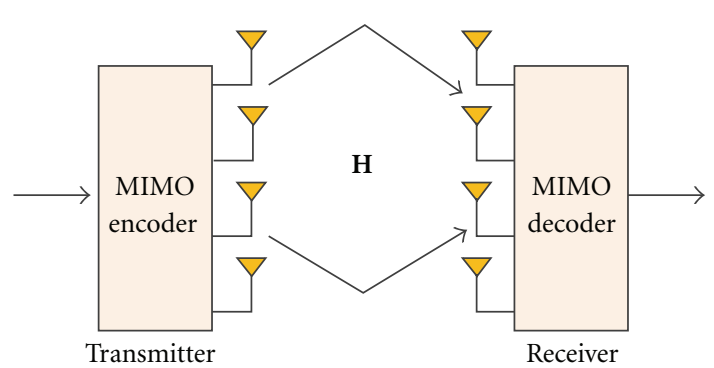

(a) Conventional MIMO system

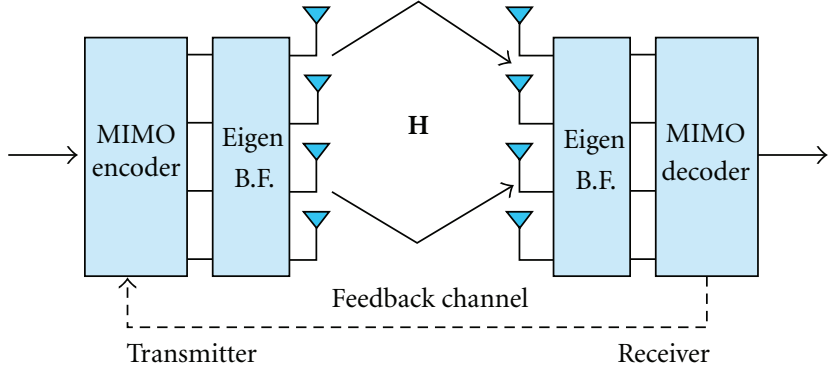

(b) Close loop beamforming

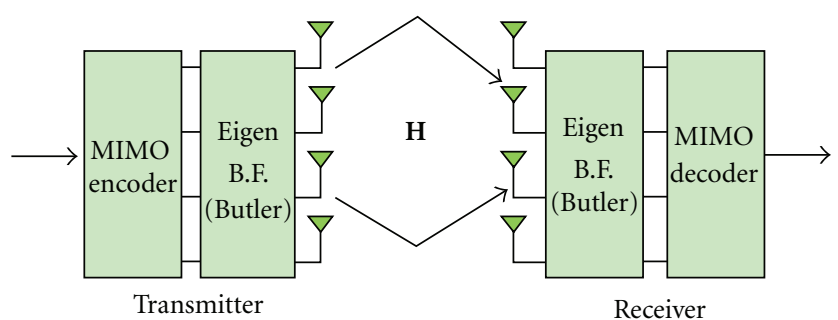

(c) Open loop beamforming

Figure 1: System overviews.

Thus, the capacity of MIMO systems can be given by

$$
C=\log _{2} \operatorname{det}\left(\mathbf{I}_{N_{r}}+\frac{P_{t}}{P_{N} N_{t}} \mathbf{H}^{a} \mathbf{H}^{a *}\right),
$$

where $\mathbf{I}_{N r}$ is the identity matrix having $N_{r} \times N_{r}$ dimension, $\mathbf{H}^{a}$ is the channel matrix having $N_{r} \times N_{t}$ dimension.

Figure 2 shows the simulated channel matrices from statistical modeling adopted by [20]. The basis for the statistical modeling of MIMO fading channels is approximated by the physical paths partitioning into angularly resolvable bins and aggregated to form resolvable paths, whose channel gains are $\mathbf{H}_{k l}^{a}$. Assuming that $a_{i}$ of the physical paths is independent. We use (4)-(7) to find channel matrix for conventional MIMO and (14)-(16) to find channel matrix for open loop beamforming.

\section{Analytical Analysis of Open Loop Beamforming}

It has been demonstrated in the literatures [22-27] that the channel capacity depends on the channel correlation. The large amplitude of correlation coefficient degrades the capacity performance. In this section, the analytical analysis of how open loop beamforming improves the channel capacity is described by showing the impact of using open loop beamforming on the channel correlation.

Let the channel matrix be modeled as

$$
\mathbf{H}=\Psi_{r}^{1 / 2} \mathbf{H}_{i i d} \boldsymbol{\Psi}_{t}^{1 / 2}
$$

where $\mathbf{H}_{i i d}$ is the complex random matrix in which its entries are identically independent distribution, $\boldsymbol{\Psi}_{r}$ and $\boldsymbol{\Psi}_{t}$ represent the matrices of channel correlation at receiver and transmitter, respectively.
Using Conventional MIMO System. According to (4), the $(k, l)$ th entry of correlation matrix at receiver is able to be given as

$$
\begin{aligned}
\left.\Psi_{r}\right|_{k, l}=E\{ & \left(\sum_{i} a_{i}^{b} e^{-j 2 \pi k \Delta_{r} \Omega_{r i}} \mathbf{e}_{t}\left(\Omega_{t i}\right)^{*}\right) \\
& \left.\times\left(\sum_{i^{\prime}} a_{i^{\prime}}^{b} e^{-j 2 \pi l \Delta_{r} \Omega_{r i^{\prime}}} \mathbf{e}_{t}\left(\Omega_{t i^{\prime}}\right)^{*}\right)^{*}\right\},
\end{aligned}
$$

where $E\{\cdot\}$ is the expectation operation. Assuming all the propagation paths are independent from each other, the correlation element in (19) can be reduced to

$$
\left.\Psi_{r}\right|_{k, l}=\sum_{i}\left|a_{i}^{b}\right|^{2} e^{-j 2 \pi(k-l) \Delta_{r} \Omega_{r i}}
$$

Same as the receiver, the $(k, l)$ th entry of correlation matrix at transmitter is given by

$$
\left.\Psi_{t}\right|_{k, l}=\sum_{i}\left|a_{i}^{b}\right|^{2} e^{j 2 \pi(k-l) \Delta_{t} \Omega_{t i}}
$$

In literatures, the degradation of channel capacity depends on the magnitude of correlation coefficient. As seen in (20) and (21), the magnitude is varied by multipath attenuation factors and angle of arrival or departure. However, in order to provide more insight on correlation coefficient, this paper assumes the Gaussian distribution for the attenuation $a_{i}$ and the uniform distribution for the directional cosine within the range of angle spread $\sigma$. As a result, the mean of correlation coefficient at receiver is given by

$$
\left.\bar{\Psi}_{r}\right|_{k, l}=\int \sum_{i}\left|a_{i}^{b}\right|^{2} e^{-j 2 \pi(k-l) \Delta_{r} \Omega_{r i}} \operatorname{prob}\left(a_{i}^{b}, \Omega_{r i}\right) d a_{i}^{b} d \Omega_{r i} .
$$




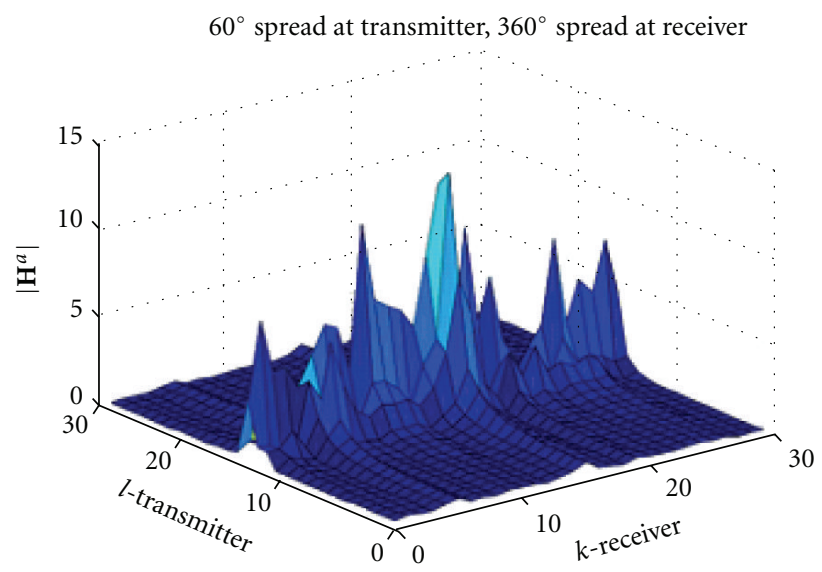

(a)

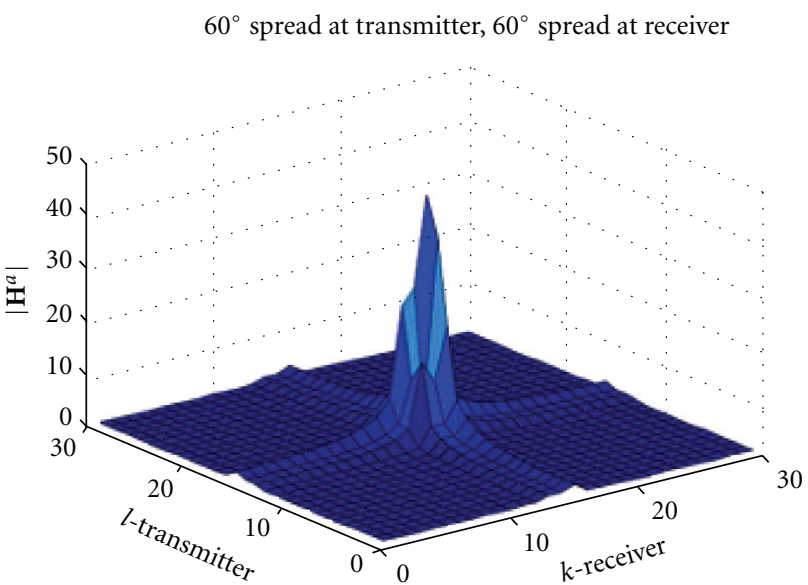

(c)

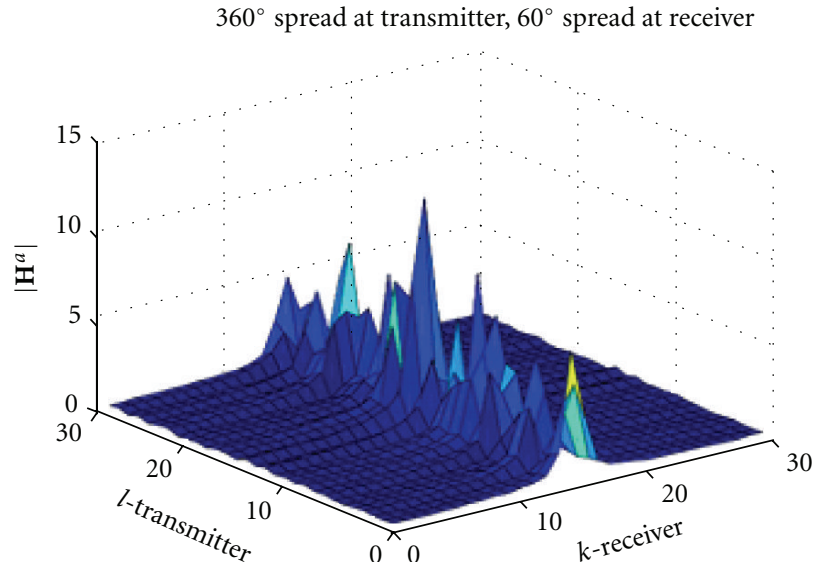

(b)

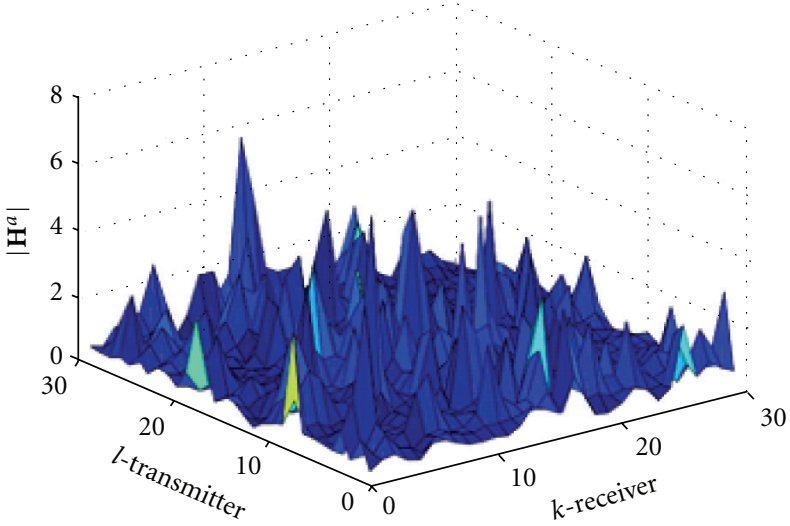

(d)

Figure 2: Examples of $\mathbf{H}^{\mathrm{a}}$ with different angle spreads at transmitter and receiver.

Because the property of each path is independent from each other and also the attenuation is independent from the directional cosine, then

$$
\left.\bar{\Psi}_{r}\right|_{k, l}=\sum_{i}\left|\bar{a}_{i}^{b}\right|^{2} e^{-j 2 \pi(k-l) \Delta_{r} \bar{\Omega}_{r i}} \frac{\sin \left(\pi(k-l) \Delta_{r} \sigma_{r i}\right)}{\pi(k-l) \Delta_{r} \sigma_{r i}},
$$

where $\bar{a}_{i}^{b}$ is the mean value of $a_{i}^{b}$ and $\bar{\Omega}_{r i}$ is the mean value of $\Omega_{r i}$

In (23), it can be noticed that the mean of correlation coefficient depends on the angle spread. This mean correlation is close to 0 when the angle spread is large. If the angle spread is very small and assuming the same mean of directional cosine for all paths, then the magnitude of mean correlation can be given by

$$
\left|\bar{\Psi}_{r}\right|=\sum_{i}\left|\bar{a}_{i}^{b}\right|^{2} .
$$

With the same derivation as receiver, the mean of correlation coefficient at transmitter is given by $\left|\bar{\Psi}_{t}\right|=$ $\sum_{i}\left|\bar{a}_{i}^{b}\right|^{2}$.
Using Open Loop Beamforming. According to (16) and (18), the correlation matrices of open loop beamforming can be formed as

$$
\mathbf{H}^{a}=\Psi_{r}^{a(1 / 2)} \mathbf{H}_{i i d} \Psi_{t}^{a(1 / 2)} .
$$

Then, the $(k, l)$ th entry of correlation matrix at the receiver is expressed by

$$
\begin{aligned}
\left.\Psi_{r}^{a}\right|_{k, l}=E & \left\{\left(\mathbf{e}_{r}\left(\frac{k}{L_{r}}\right)^{*}\left(\sum_{i} a_{i}^{b} \mathbf{e}_{r}\left(\Omega_{r i}\right) \mathbf{e}_{t}\left(\Omega_{t i}\right)^{*}\right) \mathbf{U}_{t}\right)\right. \\
& \left.\times\left(\mathbf{e}_{r}\left(\frac{l}{L_{r}}\right)^{*}\left(\sum_{i^{\prime}} a_{i^{\prime}}^{b} \mathbf{e}_{r}\left(\Omega_{r i^{\prime}}\right) \mathbf{e}_{t}\left(\Omega_{t i^{\prime}}\right)^{*}\right) \mathbf{U}_{t}\right)^{*}\right\} .
\end{aligned}
$$

Assuming all the propagation paths are independent,

$$
\left.\Psi_{r}^{a}\right|_{k, l}=\sum_{i}\left|a_{i}^{b}\right|^{2}\left(\mathbf{e}_{r}\left(\frac{k}{L_{r}}\right)^{*} \mathbf{e}_{r}\left(\Omega_{r i}\right)\right)\left(\mathbf{e}_{r}\left(\frac{l}{L_{r}}\right)^{*} \mathbf{e}_{r}\left(\Omega_{r i}\right)\right)^{*} .
$$




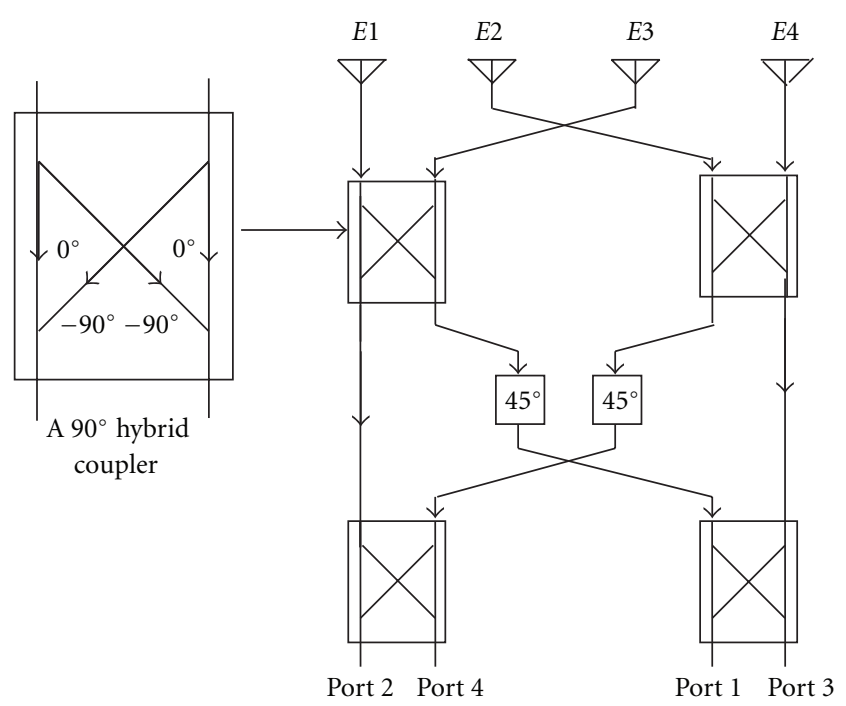

Figure 3: Block diagram of Butler matrix [18].

For the mean value of open loop beamforming, the same assumption with conventional MIMO has been used. From (26), the mean of correlation coefficient at receiver is given by

$$
\begin{aligned}
\left.\overline{\mathbf{\Psi}}_{r}^{a}\right|_{k, l}=\int \sum_{i}\left|a_{i}^{b}\right|^{2}\left(\mathbf{e}_{r}\left(\frac{k}{L_{r}}\right)^{*} \mathbf{e}_{r}\left(\Omega_{r i}\right)\right) \\
\\
\times\left(\mathbf{e}_{r}\left(\frac{l}{L_{r}}\right)^{*} \mathbf{e}_{r}\left(\Omega_{r i}\right)\right)^{*} \operatorname{prob}\left(a_{i}^{b}, \Omega_{r i}\right) d a_{i}^{b} d \Omega_{r i} .
\end{aligned}
$$

Due to the independent property, then

$$
\begin{aligned}
\left.\overline{\boldsymbol{\Psi}}_{r}^{a}\right|_{k, l}= & \sum_{i}\left|\bar{a}_{i}^{b}\right|^{2} \int \frac{1}{N_{r}} \sum_{n=0}^{N_{r}-1} e^{\left(j 2 \pi n \Delta_{r}\left(\left(k / L_{r}\right)-\Omega_{r i}\right)\right)} \frac{1}{N_{r}} \\
& \times \sum_{n^{\prime}=0}^{N_{r}-1} e^{\left(-j 2 \pi n^{\prime} \Delta_{r}\left(\left(l / L_{r}\right)-\Omega_{r i}\right)\right)} \operatorname{prob}\left(\Omega_{r i}\right) d \Omega_{r i} \\
= & \sum_{i}\left|\bar{a}_{i}^{b}\right|^{2} \frac{1}{N_{r}^{2}} \sum_{n=0}^{N_{r}-1} \sum_{n^{\prime}=0}^{N_{r}-1} e^{\left(j 2 \pi \Delta_{r}\left(\left(n k-n^{\prime} l / L_{r}\right)\right)\right)} \\
& \times e^{-j 2 \pi\left(n-n^{\prime}\right) \Delta_{r} \bar{\Omega}_{r i}} \frac{\sin \left(\pi\left(n-n^{\prime}\right) \Delta_{r} \sigma_{r i}\right)}{\pi\left(n-n^{\prime}\right) \Delta_{r} \sigma_{r i}} .
\end{aligned}
$$

For a small angle spread,

$$
\begin{aligned}
\left.\overline{\boldsymbol{\Psi}}_{r}^{a}\right|_{k, l}= & \sum_{i}\left|\bar{a}_{i}^{b}\right|^{2}\left(\frac{1}{N_{r}} \cdot \frac{1-e^{\left(j 2 \pi N_{r} \Delta_{r}\left(\left(k / L_{r}\right)-\bar{\Omega}_{r i}\right)\right)}}{1-e^{\left(j 2 \pi \Delta_{r}\left(\left(k / L_{r}\right)-\bar{\Omega}_{r i}\right)\right)}}\right) \\
& \times\left(\frac{1}{N_{r}} \cdot \frac{1-e^{\left(j 2 \pi N_{r} \Delta_{r}\left(\left(l / L_{r}\right)-\bar{\Omega}_{r i}\right)\right)}}{1-e^{\left(j 2 \pi \Delta_{r}\left(\left(l / L_{r}\right)-\bar{\Omega}_{r i}\right)\right)}}\right)^{*}
\end{aligned}
$$

$$
\begin{aligned}
= & \sum_{i}\left|\bar{a}_{i}^{b}\right|^{2} e^{\left(j \pi\left(N_{r}-1\right)(k-l) / N_{r}\right)} \\
& \times\left(\frac{\sin \left(\pi N_{r} \Delta_{r}\left(\left(k / L_{r}\right)-\bar{\Omega}_{r i}\right)\right)}{N_{r} \sin \left(\pi \Delta_{r}\left(\left(k / L_{r}\right)-\bar{\Omega}_{r i}\right)\right)}\right) \\
& \times\left(\frac{\sin \left(\pi N_{r} \Delta_{r}\left(\left(l / L_{r}\right)-\bar{\Omega}_{r i}\right)\right)}{N_{r} \sin \left(\pi \Delta_{r}\left(\left(l / L_{r}\right)-\bar{\Omega}_{r i}\right)\right)}\right) .
\end{aligned}
$$

For the transmitter, the mean of correlation coefficient can be given as

$$
\begin{aligned}
\left.\overline{\boldsymbol{\Psi}}_{t}^{a}\right|_{k, l}= & \sum_{i}\left|\bar{a}_{i}^{b}\right|^{2} e^{\left(-j \pi\left(N_{t}-1\right)(k-l) / N_{t}\right)} \\
& \times\left(\frac{\sin \left(\pi N_{t} \Delta_{t}\left(\left(k / L_{t}\right)-\bar{\Omega}_{t i}\right)\right)}{N_{t} \sin \left(\pi \Delta_{t}\left(\left(k / L_{t}\right)-\bar{\Omega}_{t i}\right)\right)}\right) \\
& \times\left(\frac{\sin \left(\pi N_{t} \Delta_{t}\left(\left(l / L_{t}\right)-\bar{\Omega}_{t i}\right)\right)}{N_{t} \sin \left(\pi \Delta_{t}\left(\left(i / L_{t}\right)-\bar{\Omega}_{t i}\right)\right)}\right) .
\end{aligned}
$$

Due to the fact that

$$
\begin{gathered}
\left(\sin \left(\pi N_{t} \Delta_{t}\left(\left(k / L_{t}\right)-\bar{\Omega}_{t i}\right)\right) / N_{t} \sin \left(\pi \Delta_{t}\left(\left(k / L_{t}\right)-\bar{\Omega}_{t i}\right)\right)\right) \\
=\left\{\begin{array}{l}
1 k / L_{t}=\Omega_{t i} \\
<1 k / L_{t} \neq \Omega_{t i} .
\end{array}\right.
\end{gathered}
$$

The magnitude of mean correlation, for $k \neq l$, at the receiver when using open loop beamforming is given by

$$
\left|\overline{\boldsymbol{\Psi}}_{r}^{a}\right|=\left|\overline{\boldsymbol{\Psi}}_{t}^{a}\right|<\sum_{i}\left|\bar{a}_{i}^{b}\right|^{2} .
$$

Comparing between (24) and (33), the magnitude of mean correlation achieved by using open loop beamforming is less than using conventional MIMO system. Therefore, according to the results in the literature [22-27], it can be implied that the capacity of MIMO system can be improved when applying open loop beamforming instead of conventional MIMO.

\section{Practical Realization Using Butler Matrix}

Figure 3 shows a block diagram of Butler matrix [18] which is applied to the concept of open loop beamforming for $4 \times 4$ MIMO systems. It is constituted by four $90^{\circ}$ hybrid couplers, 2 phase shifters $45^{\circ}$, and a crossover. The fixed beamforming matrix is a bidirectional transmission. Hence, it can be used for either receiver or transmitter.

It is easily shown that the weight vectors corresponding to each port presented in Table 1 are mutually orthogonal. Therefore, instead of using (14) and (15), the unitary matrix 


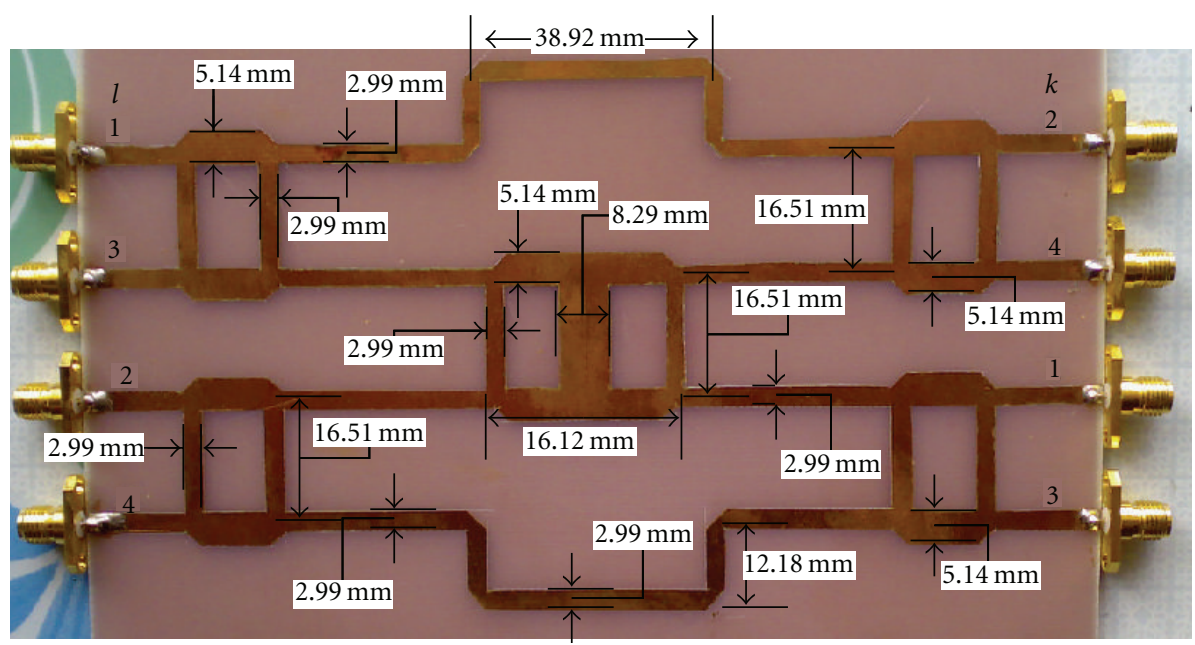

FIGURE 4: Configuration of manufactured Butler matrix.

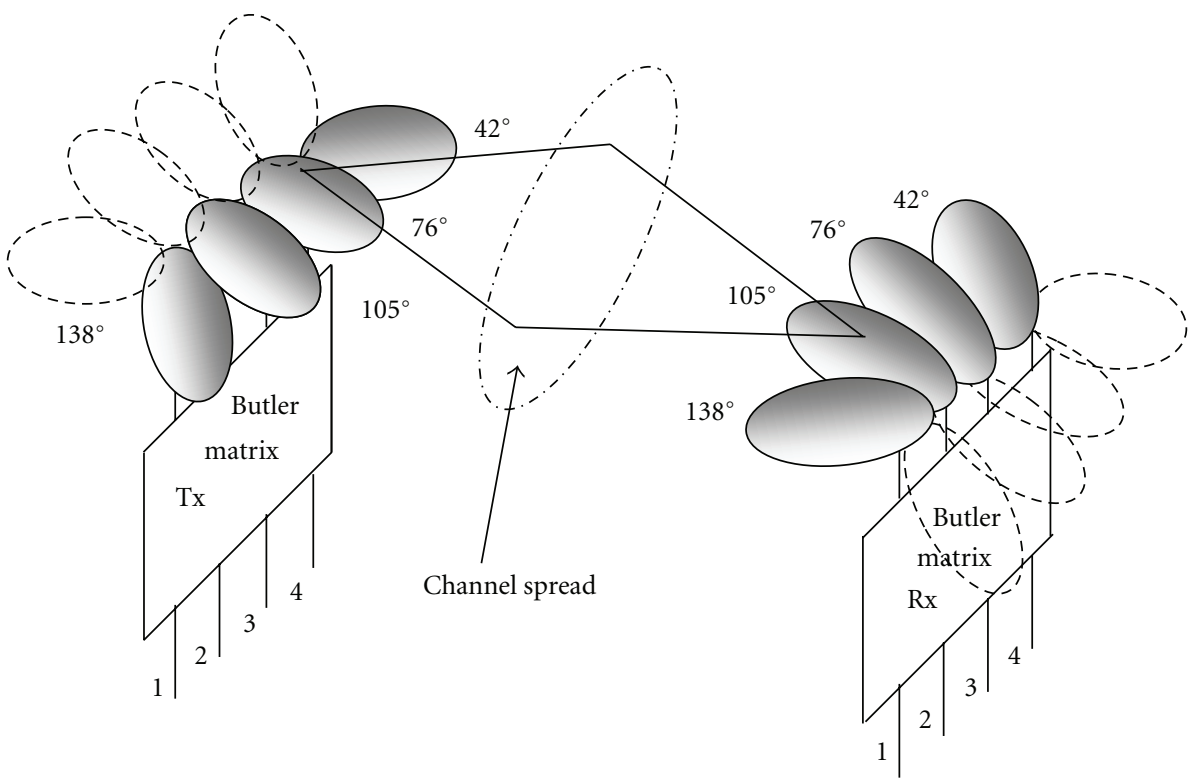

FIGURE 5: Illustration of applying Butler matrix for $4 \times 4$ MIMO systems.

TABLE 1: Element phasing, beam direction, and interelement phasing for the Butler matrix shown in Figure 3 (conceptual).

\begin{tabular}{|c|c|c|c|c|c|c|}
\hline$\theta_{k l}$ & $E 1(l=1)$ & $E 2(l=2)$ & $E 3(l=3)$ & $E 4(l=4)$ & Beam direction & Inter-element phasing \\
\hline Port $1(k=1)$ & $-45^{\circ}$ & $-180^{\circ}$ & $45^{\circ}$ & $-90^{\circ}$ & $138.6^{\circ}$ & $-135^{\circ}$ \\
\hline Port $2(k=2)$ & $0^{\circ}$ & $-45^{\circ}$ & $-90^{\circ}$ & $-135^{\circ}$ & $104.5^{\circ}$ & $-45^{\circ}$ \\
\hline Port $3(k=3)$ & $-135^{\circ}$ & $-90^{\circ}$ & $-45^{\circ}$ & $0^{\circ}$ & $75.5^{\circ}$ & $45^{\circ}$ \\
\hline Port $4(k=4)$ & $-90^{\circ}$ & $45^{\circ}$ & $-180^{\circ}$ & $-45^{\circ}$ & $41.4^{\circ}$ & $135^{\circ}$ \\
\hline
\end{tabular}

TABLE 2: Element phasing, beam direction, and interelement phasing for the Butler matrix shown in Figure 4 (manufactured).

\begin{tabular}{|c|c|c|c|c|c|c|}
\hline$\theta_{k l}$ & $E 1(l=1)$ & $E 2(l=2)$ & $E 3(l=3)$ & $E 4(l=4)$ & Beam direction & $\begin{array}{l}\text { Inter-element phasing } \\
\text { (average) }\end{array}$ \\
\hline Port $1(k=1)$ & $158^{\circ}$ & $25^{\circ}$ & $-112^{\circ}$ & $118^{\circ}$ & $138^{\circ}$ & $-130^{\circ}$ \\
\hline Port $2(k=2)$ & $-87^{\circ}$ & $-137^{\circ}$ & $176^{\circ}$ & $137^{\circ}$ & $105^{\circ}$ & $-42^{\circ}$ \\
\hline Port $3(k=3)$ & $132^{\circ}$ & $178^{\circ}$ & $-139^{\circ}$ & $-98^{\circ}$ & $76^{\circ}$ & $50^{\circ}$ \\
\hline Port $4(k=4)$ & $136^{\circ}$ & $-90^{\circ}$ & $40^{\circ}$ & $176^{\circ}$ & $42^{\circ}$ & $138^{\circ}$ \\
\hline
\end{tabular}




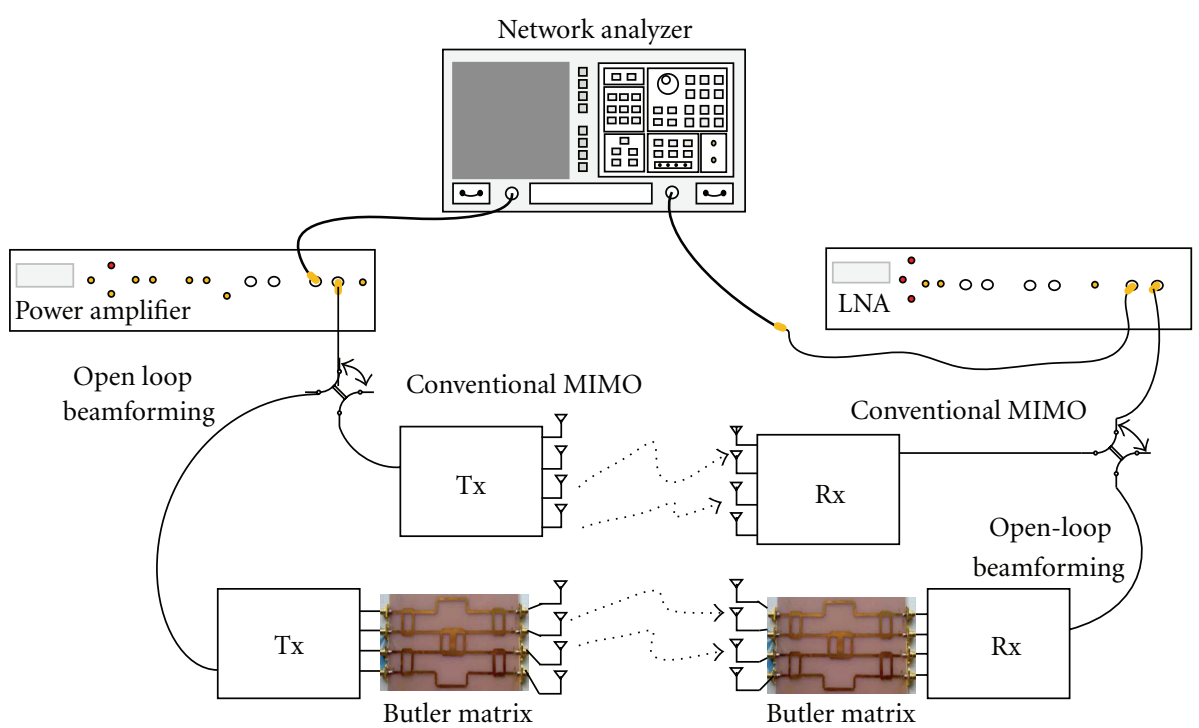

FIGURE 6: Block diagram of measurement setup.

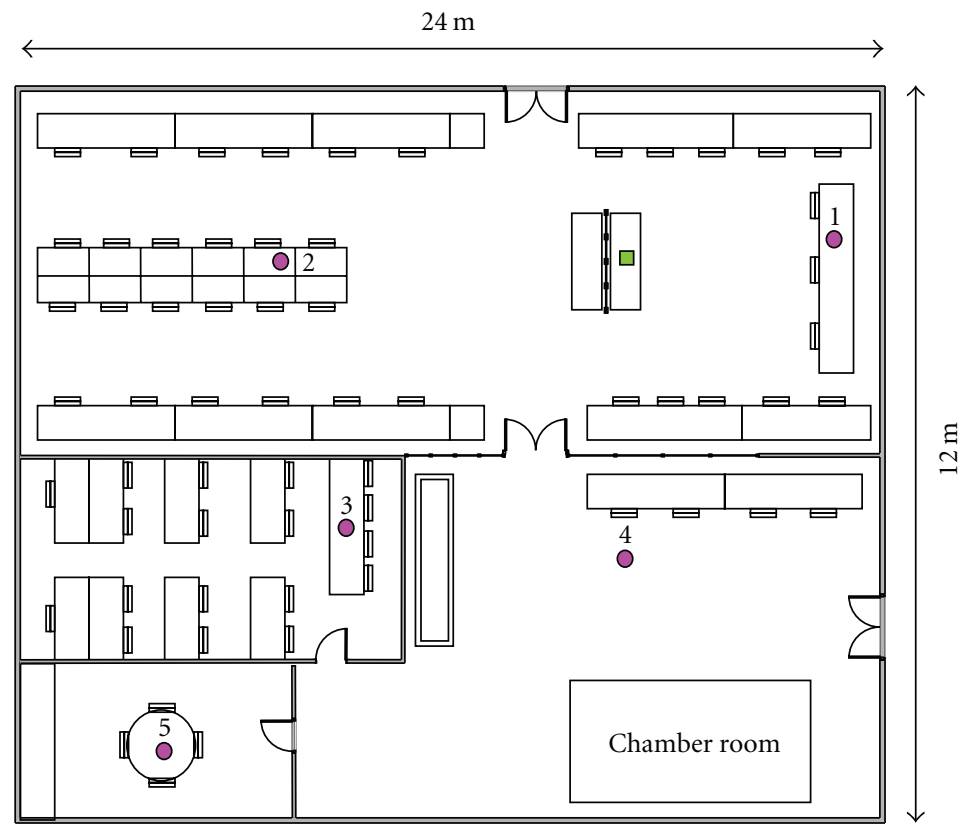

$\square$ Transmitter

○ Receiver

FIGURE 7: Measurement scenarios.

of applying Butler matrix can be written by the following expressions:

$$
\mathbf{B}_{r}=\frac{1}{\sqrt{N_{r}}}\left[\begin{array}{cccc}
e^{-j\left(-45^{\circ}\right)} & e^{-j\left(-180^{\circ}\right)} & e^{-j\left(45^{\circ}\right)} & e^{-j\left(-90^{\circ}\right)} \\
e^{-j\left(0^{\circ}\right)} & e^{-j\left(-45^{\circ}\right)} & e^{-j\left(-90^{\circ}\right)} & e^{-j\left(-135^{\circ}\right)} \\
e^{-j\left(-135^{\circ}\right)} & e^{-j\left(-90^{\circ}\right)} & e^{-j\left(-45^{\circ}\right)} & e^{-j\left(0^{\circ}\right)} \\
e^{-j\left(-90^{\circ}\right)} & e^{-j\left(45^{\circ}\right)} & e^{-j\left(-180^{\circ}\right)} & e^{-j\left(-45^{\circ}\right)}
\end{array}\right] .
$$

$$
\mathbf{B}_{t}=\frac{1}{\sqrt{N_{t}}}\left[\begin{array}{cccc}
e^{-j\left(-45^{\circ}\right)} & e^{-j\left(-180^{\circ}\right)} & e^{-j\left(45^{\circ}\right)} & e^{-j\left(-90^{\circ}\right)} \\
e^{-j\left(0^{\circ}\right)} & e^{-j\left(-45^{\circ}\right)} & e^{-j\left(-90^{\circ}\right)} & e^{-j\left(-135^{\circ}\right)} \\
e^{-j\left(-135^{\circ}\right)} & e^{-j\left(-90^{\circ}\right)} & e^{-j\left(-45^{\circ}\right)} & e^{-j\left(0^{\circ}\right)} \\
e^{-j\left(-90^{\circ}\right)} & e^{-j\left(45^{\circ}\right)} & e^{-j\left(-180^{\circ}\right)} & e^{-j\left(-45^{\circ}\right)}
\end{array}\right],
$$

Figure 4 shows a configuration of manufactured Butler matrix. The dimensions of Butler matrix can be calculated 


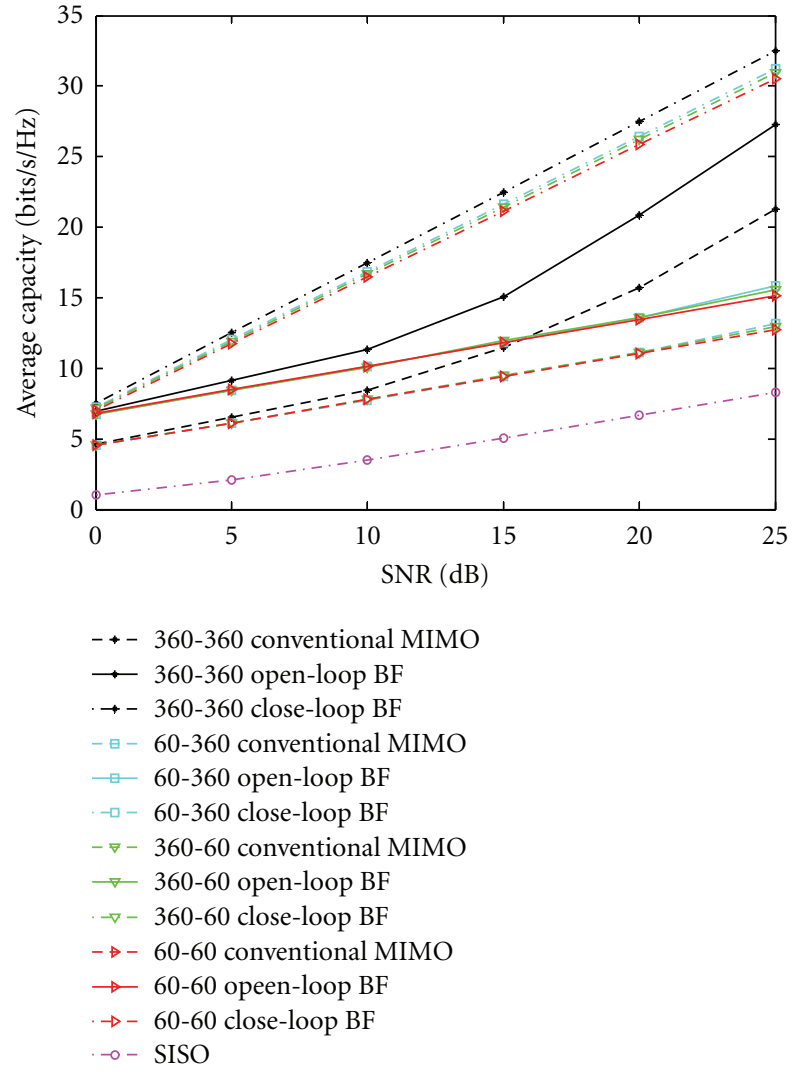

Figure 8: Average capacity (bits/s/Hz) versus SNR (dB) for 4 conditions of angle spread, $\Delta_{t}=\Delta_{r}=0.5$.

from transmission line theory. The manufactured product is also confirmed by measuring inter-element phasing and beam direction which are shown in Table 2. In this table, the distributions of all inter-element phasing are similar to conceptual Butler matrix but they are slightly deviated by \pm 10 degree. However, the beam direction is deviated by only 0.6 degree. All parameters used for experiments are presented in Table 3.

Figure 5 illustrates the beam direction of applying Butler matrix to both transmitter and receiver. It is interesting to see that the concept of open loop beamforming is successfully achieved by simply adding Butler matrices next to antenna elements. Then, the channel matrix realized by Butler matrix can be written as

$$
\mathbf{H}^{b}:=\mathbf{B}_{r}^{*} \mathbf{H B}_{t},
$$

where $\mathbf{B}_{t}$ and $\mathbf{B}_{r}$ are the unitary matrices whose columns are the basis vector in four directions for transmitter and receiver and $\mathbf{H}$ is channel matrix of size $N_{r} \times N_{t}$ to get conventional MIMO. Thus, the capacity of MIMO systems when applying Butler matrix is given by

$$
C=\log _{2} \operatorname{det}\left(\mathbf{I}_{N_{r}}+\frac{P_{t}}{P_{N} N_{t}} \mathbf{H}^{b} \mathbf{H}^{b^{*}}\right) .
$$

TABLE 3: The parameters used for measurements.

\begin{tabular}{lc}
\hline Parameters & Value \\
\hline Antenna type & Monopole \\
Number of transmitted antennas $\left(N_{t}\right)$ & 4 \\
Number of received antennas $\left(N_{t}\right)$ & 4 \\
Center frequency $\left(\lambda_{c}\right)$ & $2.4 \mathrm{GHz}$ \\
The normalized separation between the transmit & 0.5 \\
antennas $\left(\Delta_{t}\right)$ & \\
The normalized separation between receive antennas & 0.5 \\
$\left(\Delta_{r}\right)$ & \\
Distance between Tx and Rx at location 1 & $2.3 \mathrm{~m}$ \\
Distance between Tx and Rx at location 2 & $6.6 \mathrm{~m}$ \\
Distance between Tx and Rx at location 3 & $6.8 \mathrm{~m}$ \\
Distance between Tx and Rx at location 4 & $6.1 \mathrm{~m}$ \\
Distance between Tx and Rx at location 5 & $13.3 \mathrm{~m}$ \\
\hline
\end{tabular}

\section{Measurement}

Figure 6 shows a block diagram of measurement setup for $4 \times 4$ MIMO system. The network analyzer is used for measurement channel coefficients in magnitude and phase. The power amplifier (PA) is used at transmitter to provide more transmitted power. Low-noise amplifier (LNA) is used at the receiver to increase the received signal level [28]. The channel measurements are undertaken five times at each location. In each location, two modes of MIMO operation (conventional MIMO and open loop beamforming) are measured. The Butler matrices are inserted at both transmitter and receiver when measuring MIMO channels with open loop beamforming.

Figure 7 shows measurement scenarios. We choose a large room to provide various test conditions. The location of the transmitter is fixed as shown in Figure 7 with rectangular symbol. There are five measured locations for the receiver represented by circular symbol in Figure 7. It is easy to measure both conventional MIMO and open loop beamforming by using switches presented in Figure 6 . The measured results achieved by network analyzer are used as a channel response in MIMO systems. Also seen in Figure 6, apart from Butler matrix, all components of conventional MIMO and open loop beamforming are the same. Therefore, the measured channels can be directly compared to each other as presented in the next section.

\section{Results and Discussion}

6.1. Simulation Results. The simulations are undertaken by MATLAB programming, and the capacity results are evaluated by using (8), (11), and (36). For conventional MIMO approach, the channel matrix $\mathbf{H}$ is found by assumptions in (5), (6), and (7). For simulations, the channel matrix is generated by randomizing the attenuation $a_{i}$ (range from 0 to 1 ) for each path. The authors use 100 scattering paths for summation in (4). For angle of arrival/departure $\left(\phi_{r i} / \phi_{t i}\right)$, firstly the authors fix the angle spreads at transmitter and receiver. Then the angles are randomly generated within 

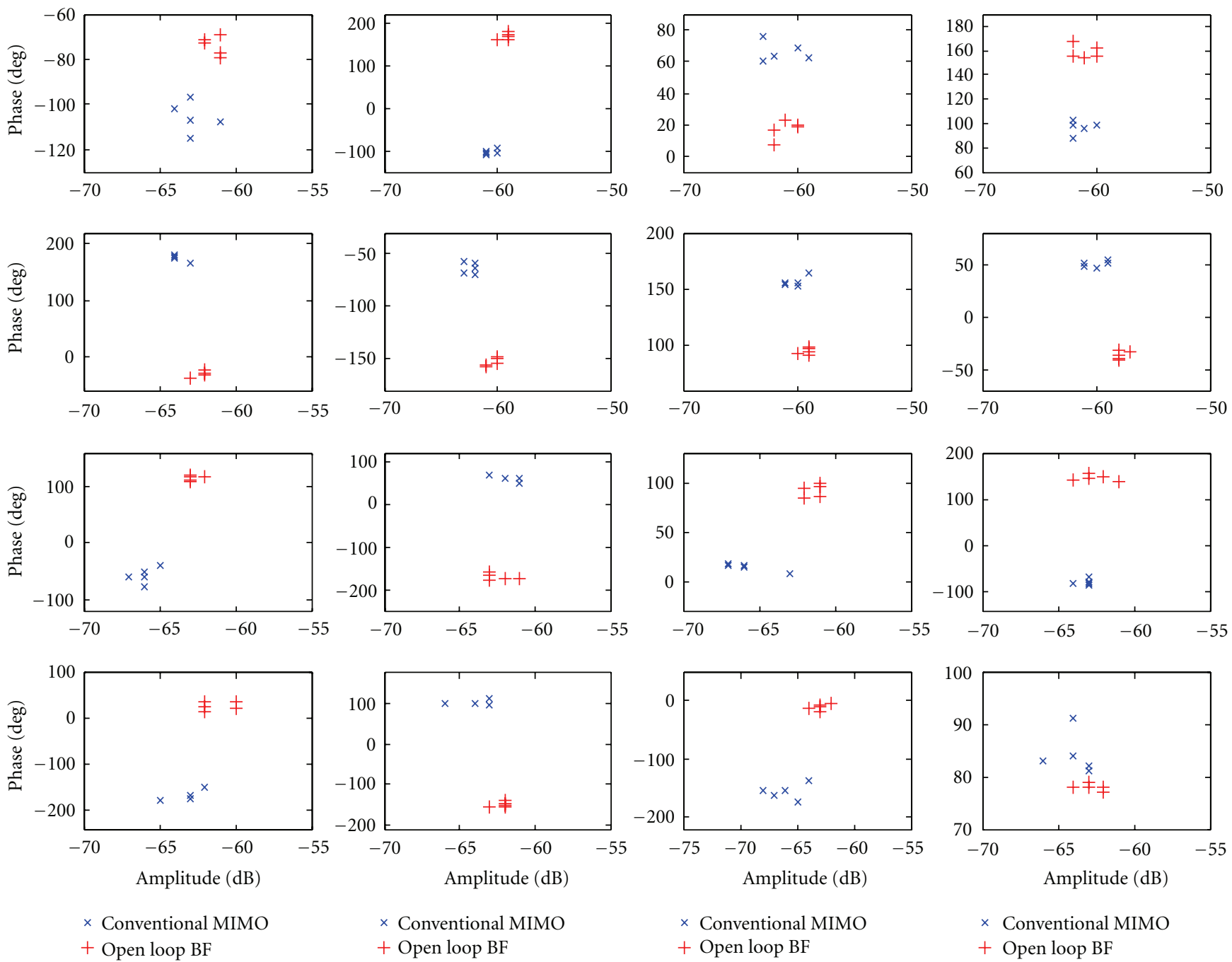

Figure 9: Measured $4 \times 4$ MIMO channels of conventional MIMO system and open loop beamforming (Butler matrix), at location 5 .

TABLE 4: Average capacity (bps/Hz) of all locations when rotating the direction of array antennas for SNR $=10 \mathrm{~dB}$.

\begin{tabular}{|c|c|c|c|c|c|c|}
\hline \multirow{3}{*}{ Location } & \multicolumn{6}{|c|}{ Direction } \\
\hline & \multicolumn{2}{|c|}{$0^{\circ}$} & \multicolumn{2}{|c|}{$45^{\circ}$} & \multicolumn{2}{|c|}{$90^{\circ}$} \\
\hline & Conv. MIMO & Open loop BF & Conv. MIMO & Open loop BF & Conv. MIMO & Open loop BF \\
\hline 1 & 8.72 & 10.12 & 9.99 & 11.68 & 11.01 & 11.59 \\
\hline 2 & 8.43 & 8.52 & 10.54 & 10.98 & 9.61 & 10.84 \\
\hline 3 & 6.46 & 6.65 & 6.88 & 9.69 & 9.67 & 10.00 \\
\hline 4 & 6.88 & 7.37 & 6.66 & 10.69 & 6.67 & 10.69 \\
\hline 5 & 10.57 & 11.03 & 11.11 & 11.52 & 11.31 & 11.59 \\
\hline
\end{tabular}

these spreads. In this paper, four cases are considered as (i) $60^{\circ}$ spread at transmitter and $360^{\circ}$ spread at receiver, denoted as $60-360$, (ii) $360^{\circ}$ spread at transmitter and $60^{\circ}$ spread at receiver, denoted as $360-60$, (iii) $60^{\circ}$ spread at transmitter and $60^{\circ}$ spread at receiver, denoted as 60-60, and (iv) $360^{\circ}$ spread at transmitter and $360^{\circ}$ spread at receiver, denoted as 360-360. Note that case (iii) is equivalent to lineof-sight scenario while case (iv) is equivalent to Rayleigh fading channel.
In Figure 8 , the average capacity comparison between $4 \times 4$ MIMO systems with conventional MIMO, open loop beamforming, close loop beamforming, and SISO system is presented. It is clearly seen that all MIMO systems offer better performance than SISO system. The results also indicatez that using the open loop beamforming realized by Butler matrix offers better performance than conventional MIMO system for all cases of angle spreads. The range of capacity enhancement is from 2 to $5 \mathrm{~dB}$ depending on characteristic 


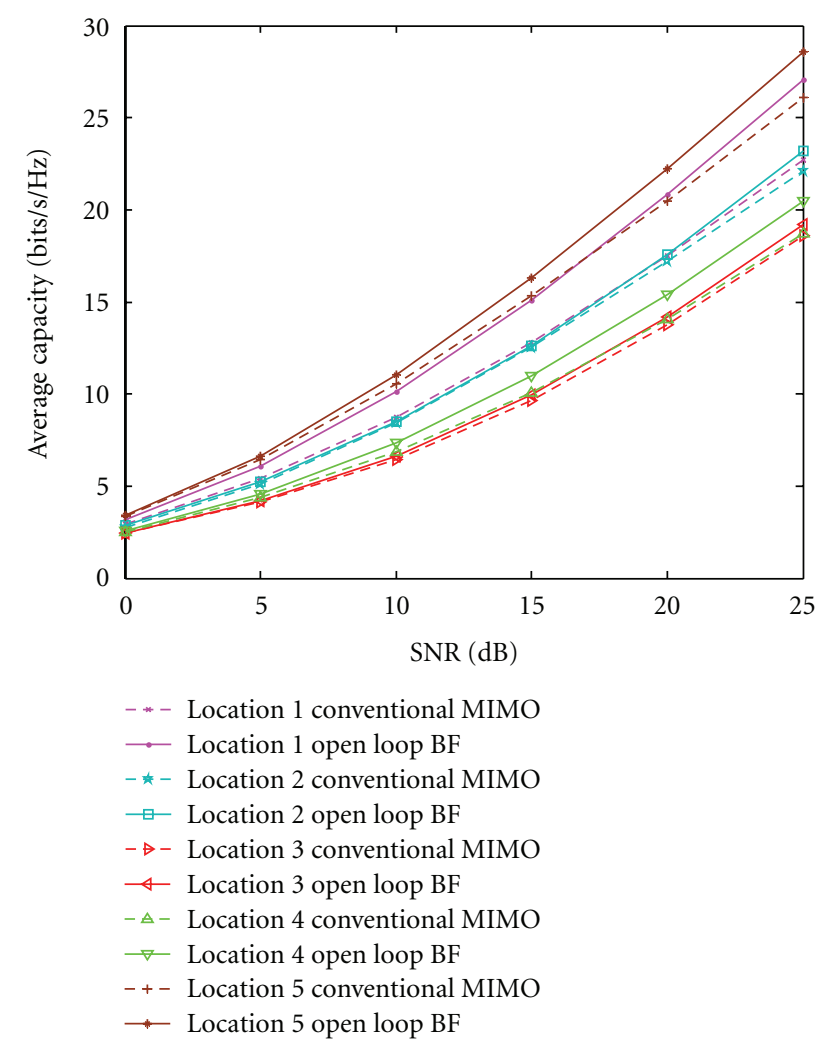

Figure 10: Average capacity (bits/s/Hz) versus SNR (dB) at each location.
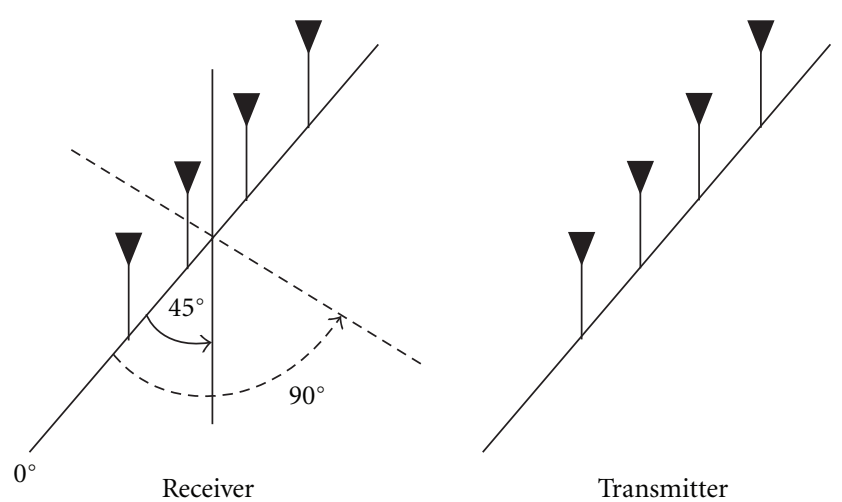

FIGURE 11: The rotating directions of array antennas for measurement scenarios.

of fadings. Although the best performance is achieved by the optimum close loop beamforming technique but it requires the full knowledge of wireless channel at the receiver. Instead of additional complexity to implement close loop beamforming, the open loop beamforming can provide the significant improvement of MIMO capacity by just inserting Butler matrices and no extra complexity is required.

6.2. Measurement Results. The channel matrices $\mathbf{H}$ and $\mathbf{H}^{b}$ are found by measured data from network analyzer. The channel fading environments are measured by changing the locations of the receiver. Five locations are considered in

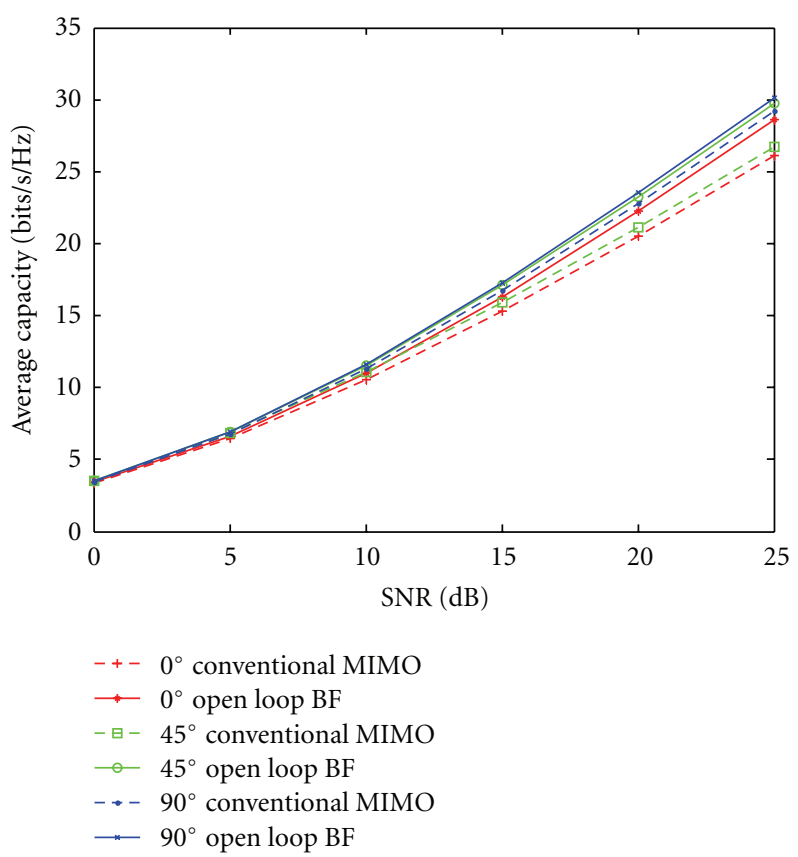

FIGURE 12: Average capacity (bits/s/Hz) versus SNR (dB) for various array directions at location 5 .

Figure 7. We also believe that the mismatches among RF circuits in transmitting/receiving components and mutual coupling effects are included in the measured channel. The simulations are undertaken by utilizing measured data into MATLAB programming and the capacity results are evaluated by using (8) and (36).

Figure 9 shows comparison of conventional and open loop beamforming channels of $4 \times 4$ MIMO systems at location 5, where $\mathbf{H}_{i j}$ is referred to the channel coefficient at $i$ th receive antenna and $j$ th transmit antenna. It can be observed that channels of conventional MIMO and open loop beamforming are relatively different. The amplitude deviation is about $\pm 5 \mathrm{~dB}$ and the phase deviation is about $\pm 100^{\circ}$. These deviations are dominant to the capacity performance of MIMO systems. For other locations, the deviations of amplitude and phase are similar to location 5 .

In Figure 10, the average capacity versus signal-to-noise ratio (SNR) at each location is presented. The results indicate that using the open loop beamforming realized by Butler matrix offers better performance than using conventional MIMO system. To compare between the simulation results presented in Figure 8 and the measurement results presented in Figure 10, the gaps between conventional MIMO and open loop beamforming in Figure 10 are different from the results in Figure 8. This is because the real propagation channel does not behave exactly as the channel modeled in simulations. However, both results provide the same agreement on improving MIMO capacity when using open loop beamforming technique.

For the measurements resulting in Figure 10, the set of transmitting and receiving antenna direction is based on face-to-face installation or $0^{\circ}$ direction depicted in Figure 11. However, the proposed open loop beamforming 
may be very sensitive to the antenna directions because both the transmitter and receiver have the fixed directivities. Accordingly, it is necessary to investigate on other array directions. The additional measurements have been done by rotating the array direction for $0^{\circ}, 45^{\circ}$, and $90^{\circ}$ degrees as depicted in Figure 11. The additional experiments are undertaken in all locations. Figure 12 shows the average capacity (bits/s/Hz) versus signal-to-noise ratio (SNR) in various directions at location 5 . The results still indicate the significant improvement of using open loop beamforming in comparson with conventional MIMO system at each array direction. In order to justify the results of other locations, the numeric values of average capacity at SNR $=10 \mathrm{~dB}$ are given in Table 4. It is obviously noticed that the benefit of open loop beamforming is pronounced for all locations and all directions. Please remember that the improvement of MIMO capacity costs the little expense of inserting Butler matrices at both transmitter and receiver without any extra complexity.

\section{Conclusion}

This paper presented the performance of MIMO systems using open loop beamforming realized by Butler matrix. The simulation results reveal that the proposed system outperforms the conventional MIMO system for every fading case. Then, this paper verified the benefit of using open loop beamforming technique for $4 \times 4$ MIMO systems by measured results. The open loop beamforming realized by Butler matrix has been implemented and compared with conventional MIMO system. The results revealed that the open loop beamforming outperforms the conventional MIMO system for all fading locations. Hence, the proposed systems are very attractive to practically implement on MIMO systems due to low cost and complexity.

\section{Acknowledgments}

This paper is financially supported by Suranaree University of Technology, Thailand and the Royal Golden Jubilee Program of Thailand Research Fund, Thailand.

\section{References}

[1] M. Bishwarup and W. H. Robert, "Performance analysis of quantized beamforming MIMO systems," IEEE Transactions on Signal Processing, vol. 54, no. 12, pp. 4753-4766, 2006.

[2] A. Sirikiat Lek, J. Zheng, O. Eric, and J. Kim, "Subspace beamforming for near-capacity MIMO performance," IEEE Transactions on Signal Processing, vol. 56, no. 11, pp. 57295733, 2008.

[3] X. Zheng, Y. Xie, J. Li, and P. Stoica, "MIMO transmit beamforming under uniform elemental power constraint," IEEE Transactions on Signal Processing, vol. 55, no. 11, pp. 5395-5406, 2007.

[4] L. Sun, M. R. McKay, and S. Jin, "Analytical performance of MIMO multichannel beamforming in the presence of unequal power Cochannel Interference and Noise," IEEE Transactions on Signal Processing, vol. 57, no. 7, pp. 2721-2735, 2009.
[5] S. L. Ariyavisitakul, E. Ojard, K. Joonsuk, Z. Jun, and N. Seshadri, "Subspace beamforming for near-capacity MIMO performance," in Proceedings of Information Theory and Applications Workshop (ITA '08), pp. 333-338, San Diego, Calif, USA, January-February 2008.

[6] R. D. Vieira, J. C. B. Brandao, and G. L. Siqueira, "MIMO measured channels: capacity results and analysis of channel parameters," in Proceedings of the International Telecommunications Symposium (ITS '06), pp. 152-157, September 2006.

[7] G. J. Foschini and M. J. Gans, "On Limits of Wireless Communications in a Fading Environment when Using Multiple Antennas," Wireless Personal Communications, vol. 6, no. 3, pp. 311-335, 1998.

[8] I. E. Telatar, Capacity of Multi-Antenna Gaussian Channels, AT\&T Bell Laboratories, Technical Memorandum, 1995.

[9] G. J. Foschini, "Layered space-time architecture for wireless communication in a fading environment when using multielement antennas," Bell Labs Technical Journal, vol. 1, no. 2, pp. 41-59, 1996.

[10] J. P. Kermoal, P. E. Mogensen, S. H. Jensen et al., "Experimental investigation of multipath richness for multi-element transmit and receive antenna arrays," in Proceedings of the 51st IEEE Vehicular Technology Conference, vol. 3, pp. 2004-2008, Spring, Tokyo, Japan, May 2000.

[11] R. Stridh, B. Ottersten, and P. Karlsson, "MIMO channel capacity on a measured indoor radio channel at $5.8 \mathrm{GHz}$," in Proceedings of the 34th Asilomar Conference on Signals, Systems and Computers, vol. 1, pp. 733-737, October 2000.

[12] A. F. Molisch, M. Steinbauer, M. Toeltsch, E. Bonek, and R. S. Thomä, "Capacity of MIMO systems based on measured wireless channels," IEEE Journal on Selected Areas in Communications, vol. 20, no. 3, pp. 561-569, 2002.

[13] L. Huang, J. W. M. Bergmans, and F. M. J. Willems, "Lowcomplexity LMMSE-based MIMO-OFDM channel estimation via angle-domain processing," IEEE Transactions on Signal Processing, vol. 55, no. 12, pp. 5668-5680, 2007.

[14] L. Huang, C. K. Ho, J. W. M. Bergmans, and F. M. J. Willems, "Pilot-aided angle-domain channel estimation techniques for MIMO-OFDM systems," IEEE Transactions on Vehicular Technology, vol. 57, no. 2, pp. 906-920, 2008.

[15] A. F. Molisch, X. Zhang, S. Y. Kung, and J. Zhang, "DFT-based hybrid antenna selection schemes for spatially correlated MIMO channels," in Proceedings of the 14th International Symposium on Personal Indoor and Movile Radio Communication, September 2003.

[16] A. Grau, J. Romeu, S. Blanch, L. Jofre, and F. De Flaviis, "Optimization of linear multielement antennas for selection combining by means of a Butler matrix in different MIMO environments," IEEE Transactions on Antennas and Propagation, no. 11, pp. 3251-3264, 2006.

[17] A. Innok, P. Uthansakul, and M. Uthansakul, Performance of MIMO Capacity Using Angle Domain Processing Realized by Butler Matrix, ECTI-CON, KhonKhen,Thailand, 2009.

[18] J. C. Liberti and J. T. S. Rappaport, Smart Antennas for Wireless Communications, IS-95 and Third Generation CDMA Applications, chapter 3, 1999.

[19] G. Bakhshi, R. Saadat, and K. Shahtalebi, "A modified two-ring reference model for MIMO mobile-to-mobile communication channels," in Proceedings of the International Symposium on Telecommunications (IST '08), pp. 409-413, August 2008.

[20] D. Tse and P. Viswanath, Fundamentals of Wireless Communication, chapter 7, Cambridge, UK, 2005. 
[21] R. G. Tsoulos, MIMO Systems Technology for Wireless Communications, Electrical Engineering \& Applied Signal Processing Series, chapter 4, 2006.

[22] Z. Xiaolin, L. Zhaowei, W. Zongxin, H. A. Suraweera, and J. Armstrong, "Capacity analysis for a distributed MIMOOFDM system in composite spatially correlated channels," in Proceedings of the 2nd International Conference on Communications and Networking in China, pp. 1116-1120, Shanghai, China, August 2007.

[23] G. Levin and S. Loyka, "On the outage capacity distribution of correlated keyhole MIMO channels," IEEE Transactions on Information Theory, vol. 54, no. 7, pp. 3232-3245, 2008.

[24] S. Jin, X. Gao, and X. You, "On the ergodic capacity of rank-1 Ricean-fading MIMO channels," IEEE Transactions on Information Theory, vol. 53, no. 2, pp. 502-517, 2007.

[25] T. Ratnarajah, "Spatially correlated multiple-antenna channel capacity distributions," IEE Proceedings: Communications, vol. 153, no. 2, pp. 263-271, 2006.

[26] M. Kang and M. S. Alouini, "Impact of correlation on the capacity of MIMO channels," in Proceedings of the International Conference on Communications (ICC '03), vol. 4, pp. 2623-2627, May 2003.

[27] S. Hyundong, Z. W. Moe, H. L. Jae, and C. Marco, "On the capacity of doubly correlated MIMO channels," IEEE Transactions on Wireless Communications, vol. 5, no. 8, pp. 2253-2264, 2006.

[28] N. Promsuvana and P. Uthansakul, "Feasibility of adaptive $4 \times 4$ MIMO system using channel reciprocity in FDD mode," in Proceedings of the 14th Asia-Pacific Conference on Communications (APCC '08), pp. 1-5, October 2008. 

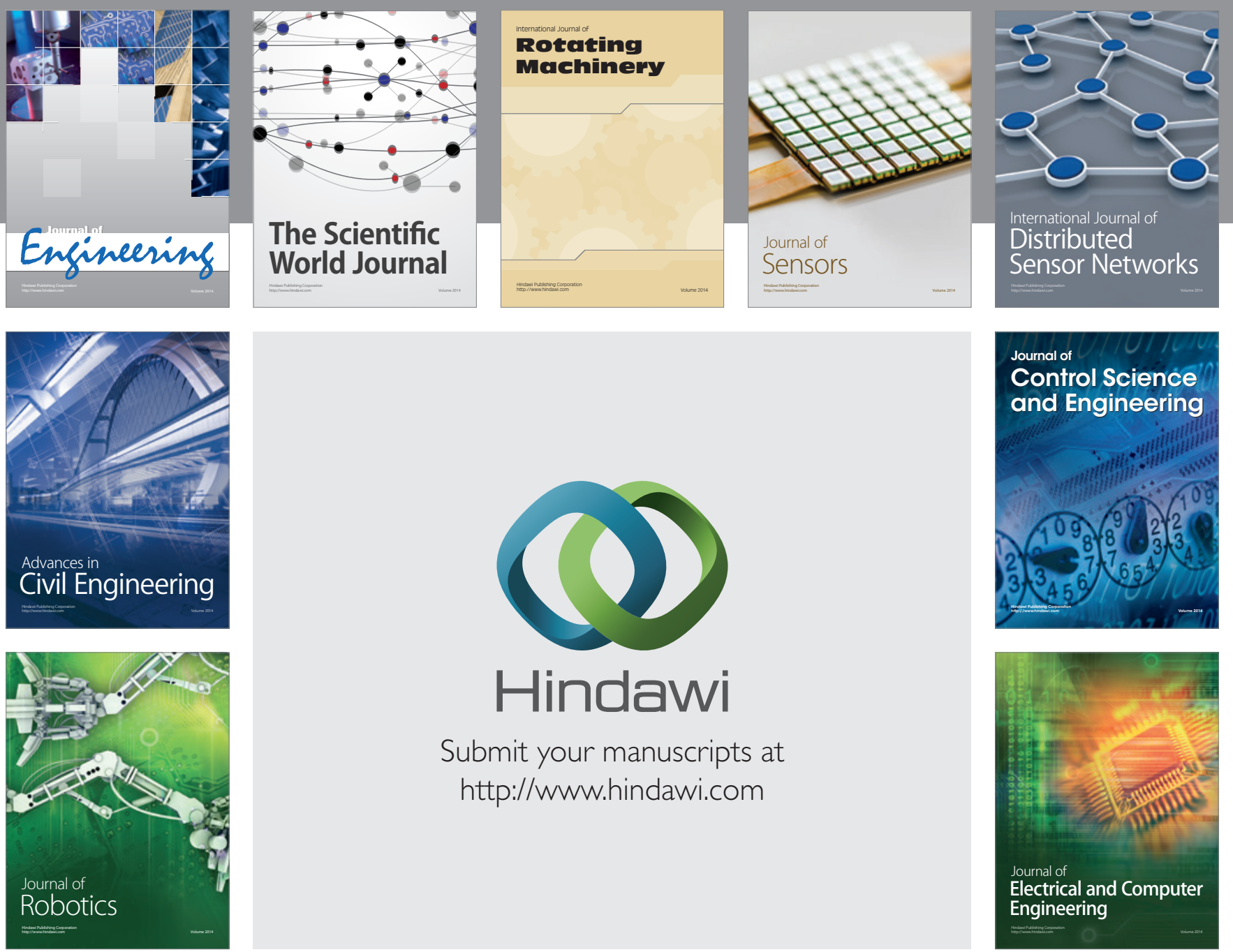

Submit your manuscripts at

http://www.hindawi.com
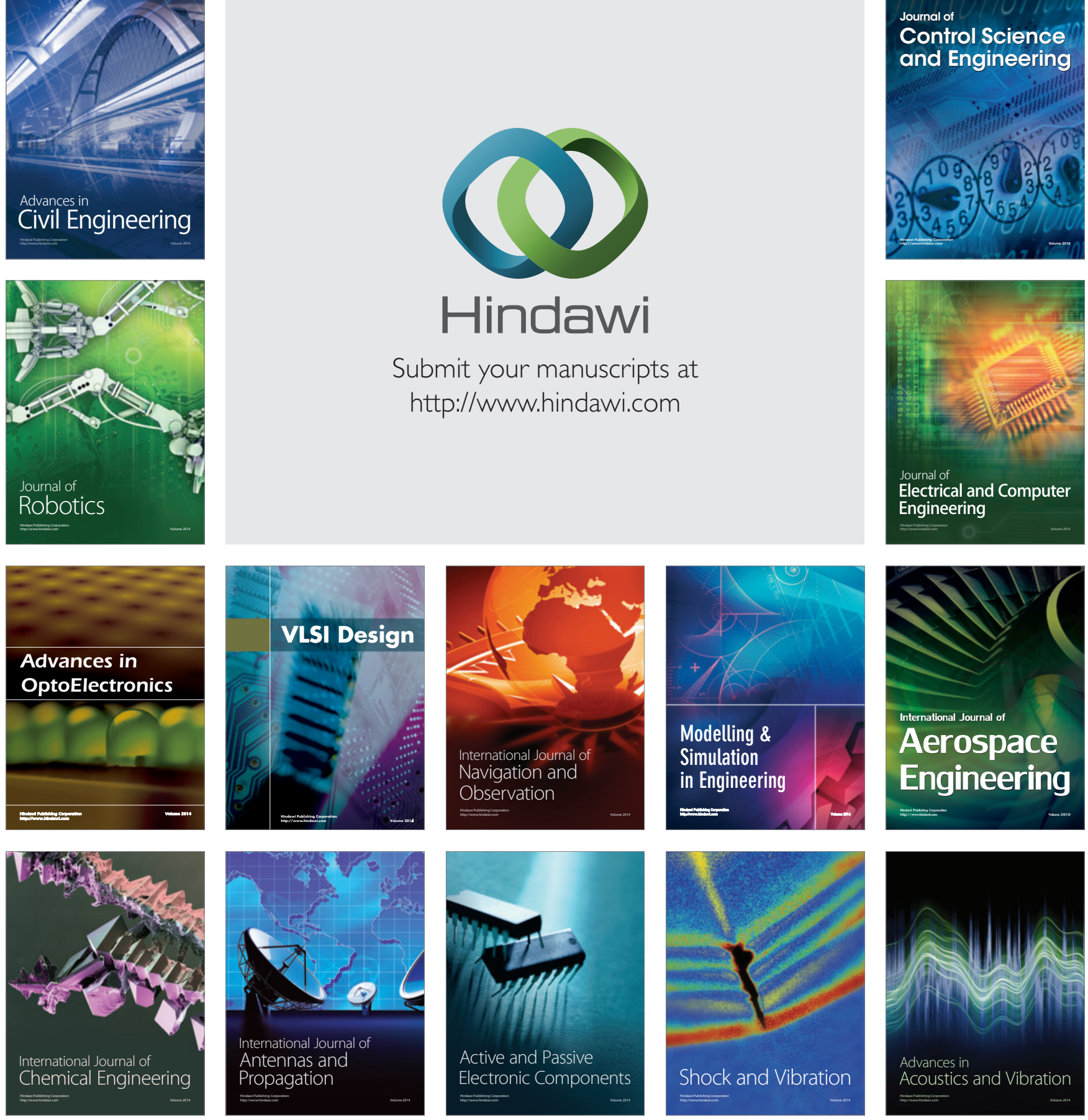